\title{
Intrarater reliability of musculoskeletal ultrasound imaging of psoas major muscle in patients with subacute low back pain and healthy controls
}

\author{
Zahra Yaghoubi ${ }^{1}$, Ismail Ebrahimi Takamjani*1미 Javad Sarrafzadeh $^{1}$, Asghar Rezasoltani², Nader Maroufi ${ }^{1}$
}

Received: 8 Apr 2019

Published: 28 Oct 2020

\section{Abstract}

Background: Psoas major (PM) is a challenging muscle from the functional and anatomical point of view. The dysfunction of this muscle can result in low back pain (LBP). This study aimed to assess the intrarater reliability of ultrasound imaging (USI) of PM muscle thickness in subacute LBP patients and healthy participants without LBP in rest and during muscle contraction conditions.

Methods: PM thickness was measured in all lumbar segments (L1-L5) using a USI device in 10 healthy and 10 subacute LBP participants. The intrarater data were assessed on the same day with 1- hour interval and after 7 days. Intraclass correlation coefficients (ICC), standard error of measurement (SEM), minimal detectable change (MDC), and independent t test were used for analyses. Significant level was set at 0.05 .

Results: PM thickness in all lumbar levels had excellent reliability (ICC range 80-98) for both groups and conditions. SEM (0.422.29) and MDC (1.16-6.34) were low, and PM thickness was greater than rest in contraction condition. There were no significant differences between the 2 groups in PM thickness.

Conclusion: The USI demonstrated good intrarater reliability for assessing PM thickness in patients with subacute LBP. The thickness of PM in patients with subacute LBP was similar with that in healthy participants.

Keywords: Reliability, Ultrasonography, Psoas major, Thickness, Low back pain

Conflicts of Interest: None declared

Funding: Iran University of Medical Sciences

*This work has been published under CC BY-NC-SA 1.0 license.

Copyright $\subseteq$ Iran University of Medical Sciences

Cite this article as: Yaghoubi Z, Ebrahimi Takamjani I, SarrafzadehJ, Rezasoltani A, Maroufi N. Intrarater reliability of musculoskeletal ultrasound imaging of psoas major muscle in patients with subacute low back pain and healthy controls. Med J Islam Repub Iran. 2020 (28 Oct);34:145. https://doi.org/10.47176/mjiri.34.145

\section{Introduction}

Low back pain (LBP) is defined as the pain felt between the costal margin and gluteal fold (1). LBP is one of the most common health problems worldwide. The cause of back pain is often unknown (2). About $80 \%$ of adults experience LBP during their life, which imposes enor-

Corresponding author: Dr Ismail Ebrahimi Takamjani, ebrahimitakamjani.e@iums.ac.ir

1. Department of Physiotherapy, School of Rehabilitation Sciences, Iran University of Medical Sciences and Health Services, Tehran, Iran

School of Rehabilitation Sciences, Shahid Beheshti university of Medical Sciences, Tehran, Iran mous direct and indirect costs on the community (2). LBP can be classified according to the duration of back pain as acute $(<4$ weeks), subacute (between 4 to 12 weeks), and chronic ( $>12$ weeks) (2). Although patients in the acute and subacute phases improve considerably (3), many pa-

$\uparrow$ What is "already known" in this topic:

Previous studies have shown that psoas major muscle is effective in developing low back pain. There is no study on the reliability of ultrasound imaging for measuring psoas major thickness in healthy individuals and patients with subacute low back pain

$\rightarrow$ What this article adds:

This study adds evidence to the reliability of the ultrasound imaging for measuring psoas major thickness in rest and contraction states in healthy individuals and patients with subacute low back pain. An interesting finding was that the thickness of psoas major was normal in patients with subacute low back pain. 
tients may experience chronic pain and disability $(4,5)$. The absence of $\mathrm{s}$ egmental and local muscle function has been suggested as a factor contributing to the high recurrence rate of $\operatorname{LBP}(6)$.

Studies indicate the dysfunction of psoas major (PM) muscle in patients with $\operatorname{LBP}(7,8)$. Regarding the selective segmental atrophy and segmental attachment of PM to lumbar spine $(9,10), \mathrm{PM}$ is involved in multiple functions in the hip and the lumbar spine (11-13). Hence, the assessment of PM using an accurate tool is essential in early phase of LBP.

The instruments provide objective and accurate information but are expensive, time- consuming, and expose patients to high radiation dose (14). Moreover, using radiological imaging (X-ray, CT, MRI) is not recommended in the acute and subacute phases of LBP (15).

The musculoskeletal ultrasound imaging (USI) is a feasible, safe, cost-effective, noninvasive, direct, and convenient method for real time assessment of muscle function in rest and during muscle contraction (16-19). Validity and reliability of USI of muscle thickness have been demonstrated in previous studies $(20,21)$. Also, a review study recommended USI for assessing muscle dysfunction in LBP (22). Thus, USI is a valuable tool to identify muscle dysfunction in LBP.

Any measurement tool must be evaluated for reliability and validity before use in the clinical and research settings. To date, no study has evaluated the reliability of USI to assess PM muscle. Therefore, the aim of the present study was to evaluate the intrarater reliability of USI in assessing PM in patients with subacute LBP and in healthy participants without LBP in rest and during muscle contraction.

\section{Methods}

\section{Participants}

An intrarater reliability study was designed. A total of 20 participants (10 healthy and 10 patients with subacute LBP) were recruited for the present study. Healthy participants had no history of LBP in the previous 2 years. Healthy participants were excluded if they had regular or professional running, cycling, professional soccer playing, and pregnancy in the previous 2 years.

The inclusion criterion for the patients with subacute LBP was unilateral back pain with or without referred leg pain, lasting between 4-12 weeks. Exclusion criteria were "red flags" for serious diseases such as spinal fracture or compressions, cauda equine syndrome, arthritis, lower extremity or spinal surgery, tumor, neuromuscular or musculoskeletal disorders, pregnancy in the previous 2 years; regular or professional training; pain aggravation during the procedure; and self-reporting rehabilitation treatment for the current episode of LBP. The study protocol was explained to all participants before testing, and written informed consents were obtained from all participants.

This protocol was approved by Iran University of Medical Sciences (IUMS), Human Research Ethics Committee (IR.IUMS.REC 1395.9211342212).

\section{Ultrasound imaging (USI) protocol}

B mode ultrasound device (Sono Ace R7, Samsung Medison, Seoul, Korea, Version 3.02) with frequency resolution of 2-8 Mega Hertz $(\mathrm{MHz})$ and curved array transducer was used to measure the thickness of PM muscle. All procedures were performed in ultrasound imaging laboratory of Iran University of Medical Sciences (IUMS).

USI was performed on the back, at the same side of the dominant hand in healthy controls, and on the painful side in subacute LBP patients. Three measurements of PM muscle thickness were performed for each participant, 2 in a single session with 1-hour interval, and 1 after 7 days. Measurements were done from L1 to L5 segmental levels of PM in rest and during contraction conditions. A trained physiotherapist performed all measurements.

The order of the measurements for groups (patients vs healthy controls), rest and contraction conditions, and segments (L1-L5) was simply randomized. For intrarater reliability, the measurements were repeated after 1 hour on the same day (for intrasession reliability) and 7 days later (for intersession reliability). Participants were asked not to do any specific exercise or training for abdomen or back muscles and not to take any analgesic drugs during the study.

In the first step, participants were exposed and then sat on a wooden chair in a relaxed state with their head and cervical spine and trunk in neutral position. In this position, the lumbar spinous processes were palpated manually and marked with a marker pen on the skin. The location of the spinous processes as described by Wallwork et al, 2007 (23), were then confirmed in parasagittal section using USI by observing the spinous processes relative to the sacrum bone as a reference point. For the contraction condition, the hands and thorax of the participants were fixed to prevent any contribution of other trunk muscles in the imaging side of hip flexion. A water-soluble transmission gel was applied to the skin over the lumbar paravertebral region for acoustic coupling. Then, the transducer was placed longitudinally (parallel to muscle fiber) $3-4 \mathrm{~cm}$ lateral and parallel to spinous process near the transverses process at each level. In the longitudinal images in every level, the acoustic shadow of the 3 consecutive transverse processes created an image which looked like the "trident sign". PM thickness was captured and measured between 2 neighboring intertransverse and 2 fascia lines, the peritoneum fascia at the bottom and the epidermis at the top at all segments (L1-L5). Recording optimal images depends on the ability to accurately identify peritoneum and skin fascia lines which are hyperechoic. Figure 1 and Figure 2 show PM thickness in rest and contraction conditions. All imaging procedures were performed by an experienced physiotherapist trained in musculoskeletal sonography.

For every level of vertebra, 3 optimal images were captured and saved in both the rest and contraction states. Optimal images were frozen at the end of the expiration phase for the rest state. Then, the imaging side of hip was flexed with knee in flexion 90 degree (hip without any rotation), such that the foot was moved to $10 \mathrm{~cm}$ above the ground level. The mean score of the 3 muscle thickness 


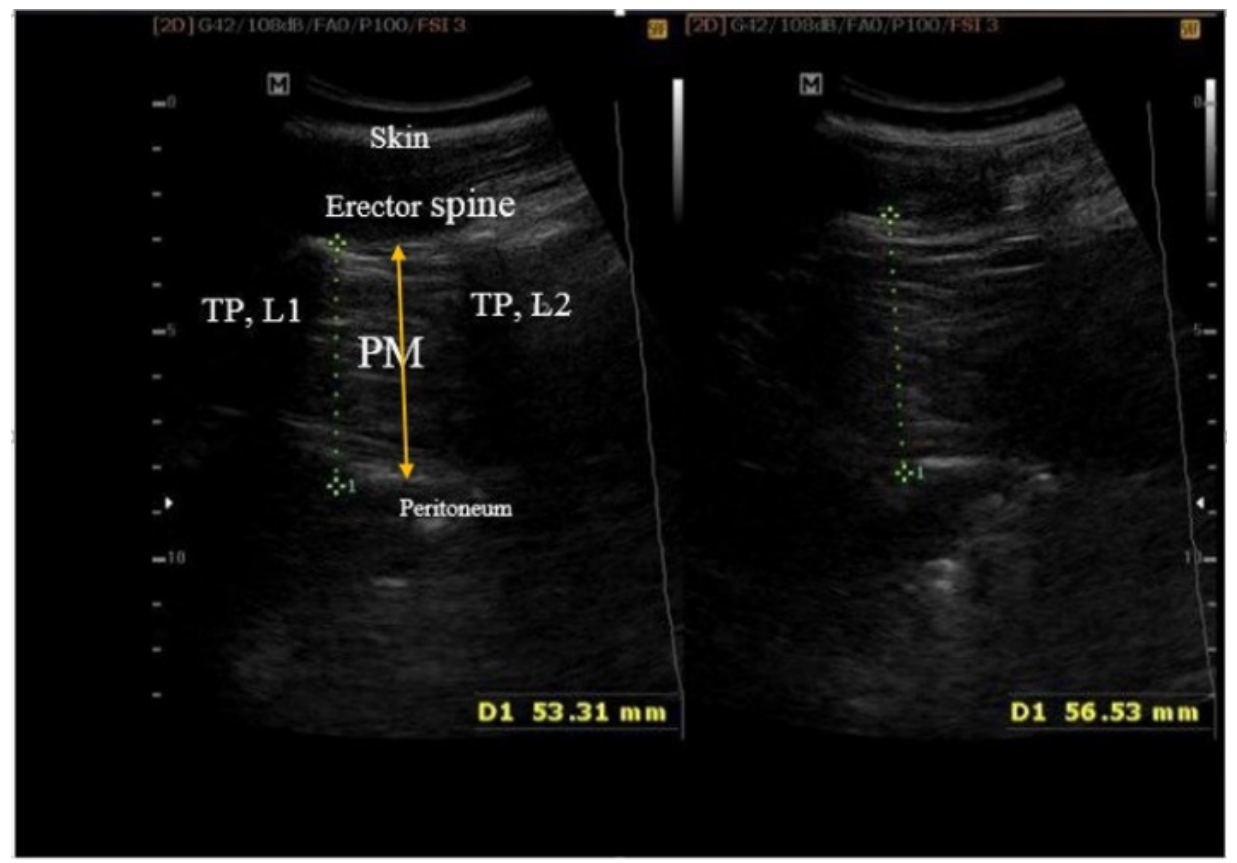

Fig. 1. The PM thicknesses in a healthy participant in rest (right) and during contraction state (left) in L2 segment. (PM, psoas major, TP: Transverses process, L1, L2 lumbar segments).

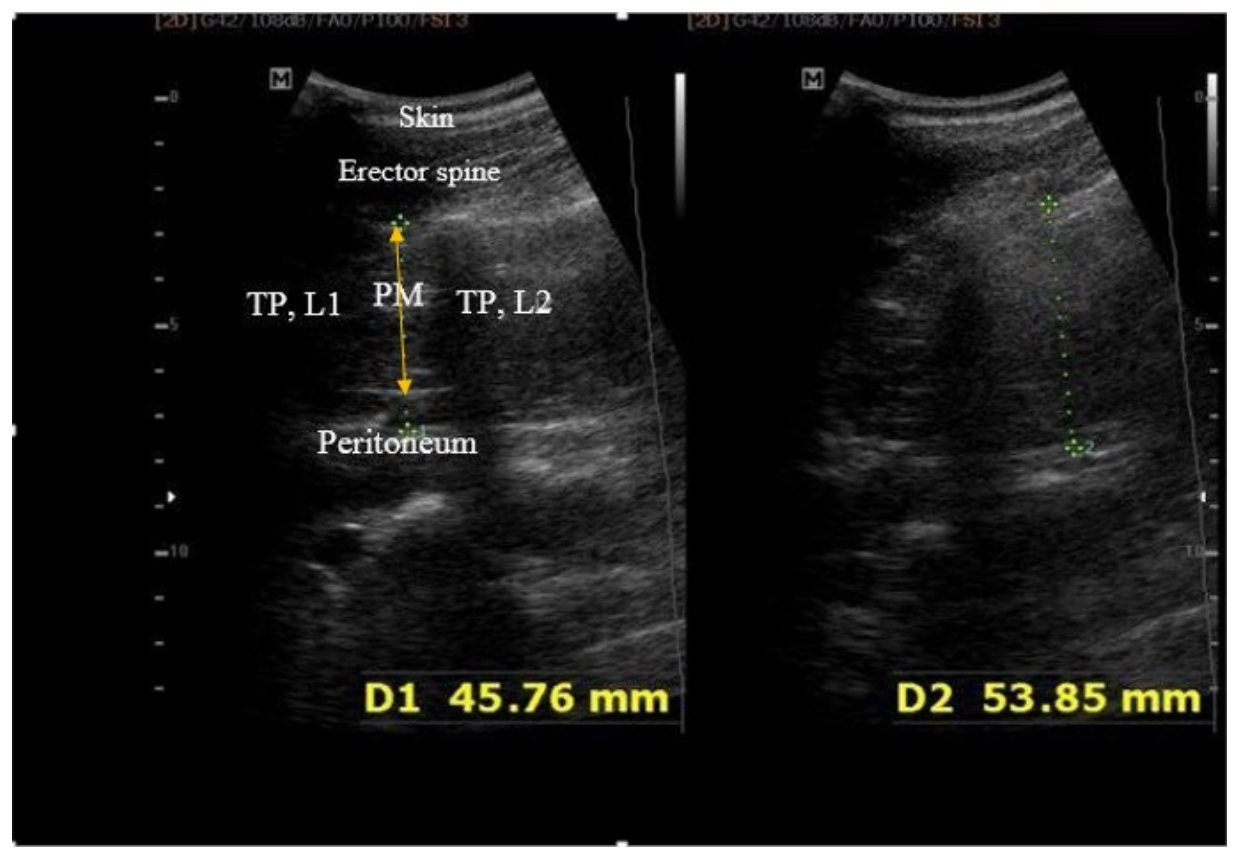

Fig. 2. PM muscle thickness in a patient with subacute low back pain in rest (right) and during contraction state (left) in L2 segment (PM, psoas major, TP: transverses process, L1, L2 lumbar segment).

measurements at each level was calculated and used as the PM thickness in that level for data analysis. Overall, 1200 images were captured (20 participants* 5 lumbar level* 2 conditions (rest and contraction) $* 2$ groups $* 3$ images at each level). All images were analyzed in offline mode with an image processing software, "Image J", (1.46r, Wayne Rasband).

\section{Statistical analysis}

Kolmogorov-Smirnov test was used to test normal distribution of data. Intraclass correlation coefficients (ICCs, 2-way random effects model, absolute agreement, and average measure) with $95 \%$ confidence interval (CI) were used for reliability analyses. Interpretation of ICC scores was as follows: fair (0.00-0.40), good (0.40-0.75), and excellent $(>0.75)(24)$. The absolute measures of reliability were calculated as standard error of measurement value with the following formula: $\mathrm{SEM}, \mathrm{SD} \times \sqrt{1-I C C}$ (25) and minimal detectable change value (MDC, $\sqrt{2} \times$ $1.96 \times \mathrm{SEM})(26)$. Independent $t$ test was used for between group comparisons of PM thickness. SPSS (Version 21.0 Chicago, IL, USA) was used for statistical analysis. Significance level was set at $\alpha=0.05$. 


\section{Results}

Patients with subacute low back pain and 10 healthy controls aged 22 to 38 years (each group: 5 males and 5 females) participated in this study. Patients had unilateral pain with mean visual analog scale (VAS) pain intensity of $5.07 \pm 1.9$. The demographic characteristics of participants are presented in Table 1. Kolmogorov-Smirnov test showed normal distribution of data.

The overall mean PM thickness of patients and healthy participants in rest and contraction was $43.42 \pm 3.19 \mathrm{~mm}$ vs $42.28 \pm 2.87 \mathrm{~mm}$ and $47.04 \pm 4.04 \mathrm{~mm}$ vs $46.91 \pm 3.13 \mathrm{~mm}$. In both patients and controls, PM thickness increased from L1 to L5 in rest (40.54-45.81 $\mathrm{mm}$ vs $39.18-45.45 \mathrm{~mm})$ and contraction (43.89-49.34 mm vs 43.67-49.62 mm). In both groups, PM thickness was significantly greater in contraction than in rest position $(p=0.001)$. The differences of PM thickness for both conditions were not statistically different between the 2 groups ( $\mathrm{p}=0.26$ ).

The ICC values observed in both groups were excellent ranging between $0.80-0.98$.

The MDC values were 3.05-3.59 mm vs $1.71-2.41 \mathrm{~mm}$ for rest and 3.62-3.77 $\mathrm{mm}$ vs $2.97-3.34 \mathrm{~mm}$ for contraction in patients and healthy controls (Table 2 and Table 3 ).
Discussion

The results of this study showed that USI protocol used in this study had excellent intra rater reliability for detecting PM thickness in healthy controls and in patients with subacute LBP in both rest and contraction for all lumbar levels of L1-L5. To our knowledge, this was the first report on reliability of ultrasound imaging for measuring psoas major thickness in patients with subacute LBP." This study showed that PM thickness was significantly greater in contraction than in rest condition and progressively increased from L1 to L5 segments. PM thickness did not show significant changes between health and patient groups. MDC values were low and nonsignificantly greater in patients compared to healthy participants.

This study found that PM thickness increased from L1 to L5 segments. The increases of PM thickness from L1 to the L5 could be due to the differences in the sizes of vertebral bodies as the vertebral bodies progressively increase in size from cervical to lumbar segments (27). We further showed no muscle thickness difference between healthy and subacute LBP groups.

This study showed that the mean thickness of PM was significantly greater in contraction than in rest position regardless of groups. Evidence indicates that PM is a fusi-

Table 1. Mean \pm SD of baseline characteristics of healthy controls and patients with subacute LBP, $\mathrm{N}=20$

\begin{tabular}{lccc}
\hline Groups & Age (years) & Weight (kg) & Height (cm) \\
\hline Healthy & $27.3 \pm 8.37$ & $65.90 \pm 5.85$ & $1.64 \pm 06$ \\
Subacute LBP & $28.3 \pm 3.71$ & $65.7 \pm 13.25$ & $1.64 \pm 09$ \\
\hline
\end{tabular}

(LBP: low back pain, BMI: body mass index, kg: kilogram. cm: centimeter).

Table 2. The mean (SD), ICC, SEM, and MDC values for PM muscle thickness in healthy controls for intrarater reliability, within session, and between days.

\begin{tabular}{|c|c|c|c|c|c|c|c|}
\hline \multicolumn{2}{|c|}{ Lumbar segments } & \multicolumn{3}{|c|}{ Within session data } & \multicolumn{3}{|c|}{ Between session data } \\
\hline L1-L5 & Mean (SD) & $\mathrm{ICC}$ & $\mathrm{SEM}(\mathrm{mm})$ & $\mathrm{MDC}(\mathrm{mm})$ & $\mathrm{ICC}$ & SEM (mm) & $\mathrm{MDC}(\mathrm{mm})$ \\
\hline L1(R) & $39.18(2.73)$ & 0.93 & 0.72 & 2.00 & 0.83 & 1.13 & 3.13 \\
\hline L1 (C) & $43.67(4.14)$ & 0.89 & 1.37 & 3.80 & 0.87 & 1.49 & 4.13 \\
\hline L2 (R) & $41.28(2.36$ & 0.96 & 0.47 & 1.31 & 0.89 & 0.78 & 2.17 \\
\hline L2 (C) & $46.36(4.13$ & 0.93 & 1.09 & 3.03 & 0.85 & 1.60 & 4.43 \\
\hline L3 ( R) & $42.38(2.43$ & 0.97 & 0.42 & 1.16 & 0.91 & 0.73 & 2.01 \\
\hline L3 (C) & $46.75(3.74)$ & 0.92 & 1.06 & 2.93 & 0.88 & 1.29 & 3.59 \\
\hline L4 (R) & $43.1(2.64)$ & 0.89 & 0.88 & 2.44 & 0.96 & 0.53 & 1.47 \\
\hline L4 (C) & $48.17(3.44)$ & 0.92 & 0.97 & 2.69 & 0.90 & 1.09 & 3.02 \\
\hline L5 (R) & $45.45(4.18)$ & 0.98 & 0.59 & 1.64 & 0.92 & 1.82 & 3.27 \\
\hline L5 (C) & $49.62(3.88)$ & 0.95 & 0.87 & 2.41 & 0.98 & 0.55 & 1.52 \\
\hline $\operatorname{Mean}(\mathrm{R})$ & $42.28(2.87)$ & 0.95 & 0.61 & 1.71 & 0.90 & 1.00 & 2.41 \\
\hline $\operatorname{Mean}(\mathrm{C})$ & $46.91(3.13)$ & 0.92 & 1.07 & 2.97 & 0.90 & 1.20 & 3.34 \\
\hline
\end{tabular}

(PM: psoas major, ICCs: intra class correlation, SEM: standard error of measurement value, MDC: minimal detectable change, L1- L5 indicates PM thickness in these lumbar segments. R: rest; C: contraction).

Table 3. The mean (SD), ICC, SEM, and MDC values for PM muscle thickness in patients with subacute low back pain for intrarater reliability, within session, and between days.

\begin{tabular}{|c|c|c|c|c|c|c|c|}
\hline \multicolumn{2}{|c|}{ Lumbar segments } & \multicolumn{3}{|c|}{ Within session data } & \multicolumn{3}{|c|}{ Between session data } \\
\hline L1-L5 & Mean (SD) & $\mathrm{ICC}$ & SEM (mm) & $\mathrm{MDC}(\mathrm{mm})$ & ICC & SEM (mm) & $\overline{\mathrm{MDC}(\mathrm{mm})}$ \\
\hline $\mathrm{L} 1(\mathrm{R})$ & $40.54(3.04)$ & 0.93 & 0.80 & 2.23 & 0.83 & 1.25 & 3.47 \\
\hline L1 (C) & $43.89(2.69)$ & 0.95 & 0.60 & 1.67 & 0.88 & 0.93 & 2.58 \\
\hline L2 (R) & $41.97(2.17)$ & 0.87 & 0.78 & 2.17 & 0.85 & 0.84 & 2.33 \\
\hline $\mathrm{L} 2(\mathrm{C})$ & $46.24(3.61)$ & 0.91 & 1.08 & 3.00 & 0.96 & 0.72 & 2.00 \\
\hline L3 (R) & $44.08(3.70)$ & 0.86 & 1.38 & 3.82 & 0.83 & 1.52 & 4.22 \\
\hline L3 (C) & $47.40(5.4)$ & 0.88 & 1.87 & 5.18 & 0.82 & 2.29 & 6.34 \\
\hline L4 (R) & $44.70(3.16)$ & 0.87 & 1.14 & 3.16 & 0.80 & 1.41 & 3.91 \\
\hline L4 (C) & $48.34(3.72)$ & 0.91 & 1.12 & 3.09 & 0.87 & 1.34 & 3.71 \\
\hline L5 (R) & $45.81(3.88)$ & 0.87 & 1.40 & 3.87 & 0.86 & 1.45 & 4.02 \\
\hline L5 (C) & $49.34(4.8)$ & 0.85 & 1.85 & 5.15 & 0.90 & 1.52 & 4.20 \\
\hline Mean (R) & $43.42(3.19)$ & 0.88 & 1.10 & 3.05 & 0.83 & 1.29 & 3.59 \\
\hline Mean (C) & $47.04(4.04)$ & 0.90 & 1.30 & 3.62 & 0.89 & 1.36 & 3.77 \\
\hline
\end{tabular}

(PM: psoas major, ICCs: intra class correlation, SEM: standard error of measurement value, MDC: minimal detectable change, L1- L5 indicates PM thickness in these lumbar segments. R: rest; C: contraction). 
form muscle and as muscle fiber shortens during low force contraction (eg, isometric contraction), muscle thickness increases (28). The finding of this study is in line with another study (29) that showed USI is able to detect lowlevel isometric contraction, including PM muscle (30).

Findings of this study demonstrated no changes in muscle thickness in patients compared to healthy controls. One explanation for this finding may be that LBP is a multidimensional condition, and there is clear difference between healthy individuals and subgroups of patients with LBP depending on duration of back pain. In fact, the impaired movement control in subacute LBP is significantly less than chronic LBP; no significant changes were reported in patients with subacute LBP compared to patients with acute episode (31). Findings indicated no atrophy of PM thickness in subacute patients, despite impaired motor control.

The ICC values were excellent in both groups. One possible reason could be from the protocol and sitting position used for measurements that was comfortable for both the participants and the examiner. US probe positioning was appropriate to capture the images accurately. There were no previous investigations with which to compare our reliability findings.

Calculation of SEM and MDC can help to detect real changes from random measurement errors. The MDC value indicates minimum amount of change, improvement or deterioration to be considered as a real change (32). Any changes must be above the MDC value to be defined as a real change. All MDCs found in this study were low. In patients with LBP, MDC values were greater than those of healthy participants. There are no previous reports in the literature regarding the MDC of PM thickness for comparison.

This study recruited young participants which decreases the generalizability of the findings. In the present study, intrarater reliability was evaluated; however, interrater reliability must be evaluated as well. Future investigations with multiple raters are warranted as different results may occur.

\section{Conclusion}

The results of this study showed that the USI protocol used in this study had excellent intrarater reliability for measuring PM thickness in patients with subacute LBP and healthy controls in both rest and contraction states for all lumbar levels.

\section{Acknowledgments}

The authors would like to thank Iran University of Medical Sciences (IUMS) for supporting this project.

\section{Conflict of Interests}

The authors declare that they have no competing interests.

\section{References}

1. Krismer M, Van Tulder M. Low back pain (non-specific). Best Pract Res Clin Rheumatol. 2007;21(1):77-91.

2. Brennan GP, Fritz JM, Hunter SJ, Thackeray A, Delitto A, Erhard RE.
Identifying subgroups of patients with acute/subacute "nonspecific" low back pain: results of a randomized clinical trial. Spine. 2006;31(6):623-31.

3. Qaseem A, Wilt TJ, McLean RM, Forciea MA. Noninvasive treatments for acute, subacute, and chronic low back pain: a clinical practice guideline from the American College of Physicians. Ann Intern Med. 2017;166(7):514-30.

4. Koes B, Van Tulder M, Thomas S. Diagnosis and treatment of low back pain. Bmj. 2006;332(7555):1430-4.

5. Pengel LH, Herbert RD, Maher CG, Refshauge KM. Acute low back pain: systematic review of its prognosis. Bmj. 2003;327(7410):323.

6. Hides JA, Richardson CA, Jull GA. Multifidus muscle recovery is not automatic after resolution of acute, first-episode low back pain. Spine. 1996;21(23):2763-9.

7. Makino T, Hosono N, Mukai Y, Miwa T, Fuji T. Ipsilateral atrophy of the psoas major muscle in patients with lumbar disc herniation. Clin Orthop Surg. 2009;44(2):167-72.

8. Barker KL, Shamley DR, Jackson D. Changes in the cross-sectional area of multifidus and psoas in patients with unilateral back pain: the relationship to pain and disability. spine J. 2004;29(22):E515-E9.

9. Gibbons SGT. A Hypothetical Link Between Psychosocial Factors, Pain and Sensory Motor Function using a Biomechanical Model of Psoas Major: ( MScThesis ), University of Surrey. ; 2004.

10. Bogduk N, Pearcy M, Hadfield G. Anatomy and biomechanics of psoas major. Clin Biomech (Bristol, Avon). 1992;7(2):109-19.

11. Jeon IC, Kwon OY, Weon JH, Choung SD, Hwang UJ. Comparison of psoas major muscle thickness measured by sonography during active straight leg raising in subjects with and without uncontrolled lumbopelvic rotation. Man Ther. 2016;21:165-9.

12. Yoshio M, Murakami G, Sato T, Sato S, Noriyasu S. The function of the psoas major muscle: passive kinetics and morphological studies using donated cadavers. J Orthop Sci. 2002;7(2):199-207.

13. Penning L. Psoas muscle and lumbar spine stability: a concept uniting existing controversies. Eur Spine J. 2000;9(6):577-85.

14. Snijder M, Visser M, Dekker J, Seidell J, Fuerst T, Tylavsky F, et al. The prediction of visceral fat by dual-energy X-ray absorptiometry in the elderly: a comparison with computed tomography and anthropometry. Int J Obes (Lond). 2002;26(7):984.

15. Goertz M, Thorson D, Bonsell J, Bonte B, Campbell R, Haake B, et al. Adult acute and subacute low back pain. Institute for Clinical Systems Improvement [Internet] Updated November. 2012:10-2.

16. Nestorova R, Vlad V, Petranova T, Porta F, Radunovic G, Micu MC, et al. Ultrasonography of the hip. Med Ultrason. 2012;14(3):217.

17. Takai Y, Katsumata Y, Kawakami Y, Kanehisa H, Fukunaga T. Ultrasound method for estimating the cross-sectional area of the psoas major muscle. Med Sci Sports Exerc. 2011;43(10):2000-4.

18. Harcke H, Grissom L, Finkelstein M. Evaluation of the musculoskeletal system with sonography. AJR Am J Roentgenol. 1988;150(6):1253-61.

19. Laycock J, Jerwood D. Pelvic floor muscle assessment: the PERFECT scheme. Physiotherapy. 2001;87(12):631-42.

20. Watanabe K, Miyamoto K, Masuda T, Shimizu K. Use of ultrasonography to evaluate thickness of the erector spinae muscle in maximum flexion and extension of the lumbar spine. Spine J. 2004;29(13):1472-7.

21. Rezasoltani A. The applicability of muscle ultrasonography in physiotherapy researches. J Phys Ther Sci. 2003;15(1):33-7.

22. Heidari P, Farahbakhsh F, Rostami M, Noormohammadpour P, Kordi R. The role of ultrasound in diagnosis of the causes of low back pain: a review of the literature. Asian J Sports Med. 2015;6(1).

23. Wallwork TL, Hides JA, Stanton WR. Intrarater and interrater reliability of assessment of lumbar multifidus muscle thickness using rehabilitative ultrasound imaging. J Orthop Sports Phys Ther. 2007;37(10):608-12.

24. Rosner B. Fundamentals of Biostatistics: Belmont, CA: Duxbury Press; 2006.

25. Denegar CR, Ball DW. Assessing reliability and precision of measurement: an introduction to intraclass correlation and standard error of measurement. J Sport Rehabil. 1993;2(1):35-42.

26. Bland JM. Minimal detectable change. Phys Ther Sport. 2009;10(1):39.

27. Zhou S, McCarthy I, McGregor A, Coombs R, Hughes S. Geometrical dimensions of the lower lumbar vertebrae-analysis of data from digitised CT images. Eur Spine J. 2000;9(3):242-8.

28. Azizi E, Brainerd EL, Roberts TJ. Variable gearing in pennate 
Psoas major thickness reliability

muscles. Proc Natl Acad Sci. 2008;105(5):1745-50.

29. Hodges P, Pengel L, Herbert R, Gandevia SJM, nerve. Measurement of muscle contraction with ultrasound imaging. Muscle Nerve. 2003;27(6):682-92.

30. Norasteh A, Ebrahimi E, Salavati M, Rafiei J, Abbasnejad E. Reliability of B-mode ultrasonography for abdominal muscles in asymptomatic and patients with acute low back pain. J Bodyw Mov Ther. 2007;11(1):17-20.

31. Luomajoki H, Kool J, De Bruin ED, Airaksinen OJBmd. Movement control tests of the low back; evaluation of the difference between patients with low back pain and healthy controls. BMC Musculoskelet Disord. 2008;9(1):170.

32. Fritz SL, Blanton S, Uswatte G, Taub E, Wolf SLJN, repair $n$ Minimal detectable change scores for the Wolf Motor Function Test. Neurorehabil Neural Repair. 2009;23(7):662-7. 